Classification

Physics Abstracts

$91.60 \mathrm{~B}-47.60-02.70$

\title{
Permeability of a Single Fracture; Validity of the Reynolds Equation
}

\author{
V.V. Mourzenko( $\left.{ }^{*}\right)$, J.-F. Thovert, and P.M. Adler \\ LPTM/CNRS, Asterama 2, Avenue du Téléport, 86360 Chasseneuil du Poitou, France
}

(Received 17 October 1994, received in final form 1 December 1994, accepted 7 December 1994)

\begin{abstract}
Single fractures are generated by statistical methods and described by a probability density of the profile heights and a spatial covariance function which is either Gaussian or self-affine. The results of numerical simulations baised on both 2D Reynolds and 3D Stokes equations are presented. Total fluxes predicted by these equations for the same fractures are given and discussed. The difference between predictions of both models is analysed for the range of correlation lengths and apertures of real fractures, and it is found that the total fluxes for the same fractures may differ more than two times. When the local distance between the surfaces is used, the Reynolds equation provides much better results.
\end{abstract}

\section{Introduction}

Fluid flow through systems of interconnected fractures is often the main mechanism of fluid and solute transport in low-permeable rocks and is a subject of ongoing interest in petroleum engineering and hydrogeology. Real fractures are characterized by very heterogeneous structures of open space and it is important to correctly describe their hydraulic properties.

Numerical simulations of fluid flow in a single fracture are usually based on lubrication theory, and use the Reynolds equation for calculating the pressure distribution with a "cubic law" for the flux [1-3]

$$
\begin{aligned}
\nabla \cdot\left(\frac{b^{3}(x, y)}{12 \mu} \nabla p\right)=0 \\
Q_{x}=-L_{y} \frac{b^{3}}{12 \mu} \frac{\partial p}{\partial x}
\end{aligned}
$$

where $b$ is the fracture aperture, $p$ the fluid pressure, $\mu$ the viscosity and $L_{y}$ the width of the fracture.

(*) Permanent address: Institute for Problems in Mechanics, Russian Academy of Sciences, 101, prosp Vernadskogo, Moscow, 117526, Russia. 
The Reynolds equation can be derived from the Navier-Stokes equations for low velocity and a slowly varying aperture. It is difficult to estimate analytically the validity of the lubrication approximation and only a few quantitative comparisons are available. Using the results of $[4,5]$, Zimmerman et al. [3] have found that the predictions of lubrication theory and of Stokes equations for the mean flux will not differ by more than $10 \%$ if the wavelength of the aperture oscillations exceeds $5 \sigma_{h}$, where $\sigma_{h}$ is the standard deviation of the profile height distribution. Another approach was used by Brown [2], with numerical solutions of Reynolds equation; the local ratio $r$ of the velocity gradients parallel and perpendicular to the plane of the fracture was estimated; $r$ should be small for the lubrication theory to be valid; $r$ was found to be smaller than 0.1 if the wavelength of the aperture oscillations is larger than $50 \sigma_{h}$; hence, the possible applications of Reynolds equation to natural fractures would seem to be severely restricted.

The major purpose of this work is to provide systematic results of numerical simulations based on the 2D Reynolds and 3D Stokes equations solved for the same fractures and to compare them. To the best of our knowledge, this has never been done. Fractures are described by a probability density of the profile height and by a correlation function. In this framework, two major classes of fractures are analysed, namely fractures with Gaussian or self-affine correlation functions; for sake of brevity, such fractures will be called Gaussian or self-affine. Two tentative definitions of the aperture used in the Reynolds equation (1) are given; in the first case, $b$ is simply the vertical separation between the upper and lower surfaces; in the second case, the aperture $b$ is replaced by an estimate of the actual distance between the two surfaces Permeabilities derived by the full Stokes equations and by the Reynolds equation based on $b$ and $b_{\mathrm{d}}$ are systematically given as functions of the geometrical parameters.

It is emphasized that substantial differences between predictions of the exact Stokes equations and of the Reynolds equation based on $b$, are obtained for the range of correlation lengths and apertures of real fractures, for Gaussian and self-affine surfaces. However, when the aperture $b_{\mathrm{d}}$ is used in the Reynolds equation, the comparison is significantly improved.

This comparison is important for a precise determination of permeability. However, it may have also important consequences in other domains such as dispersion of solutes in fractures; the local distribution of the fluid velocity may substantially influence the determination of the dispersion tensor $[6,7]$.

\section{Generation of Fractures}

The two surfaces of a fracture are described by the heights $z=h^{ \pm}(x, y)$ above an arbitrary reference plane $z=0$ (Fig 1). Usually, $h^{ \pm}$are random functions; they can be characterized by the two probability densities $\varphi(Z)$ and $\varphi(w)$ of the mean surface $Z=\left(h^{+}+h^{-}\right) / 2$ and of the distance $w=h^{+}-h^{-}$which are often assumed to be Gaussian

$$
\varphi(F)=\frac{1}{\sqrt{2 \pi} \sigma_{F}} \exp \left[-\frac{(F-\langle F\rangle)^{2}}{2 \sigma_{F}^{2}}\right], \quad F=Z, w, h
$$

where $\sigma_{F}^{2}$ denotes the variance $\left\langle\left(F-\langle F>)^{2}\right\rangle\right.$. The brackets correspond to the statistical average.

The spatial correlations of the fields $h^{+}$and $h^{-}$are described by the covariance functions $C_{Z}(r)$ and $C_{W}(r)$, which are defined by

$$
\begin{aligned}
C_{F}(r) & =\langle\{F(x, y)-<F>\}\{F(x+\Delta x, y+\Delta y)-<F>\}\} \\
r & =\left(\Delta x^{2}+\Delta y^{2}\right)^{1 / 2}
\end{aligned}
$$




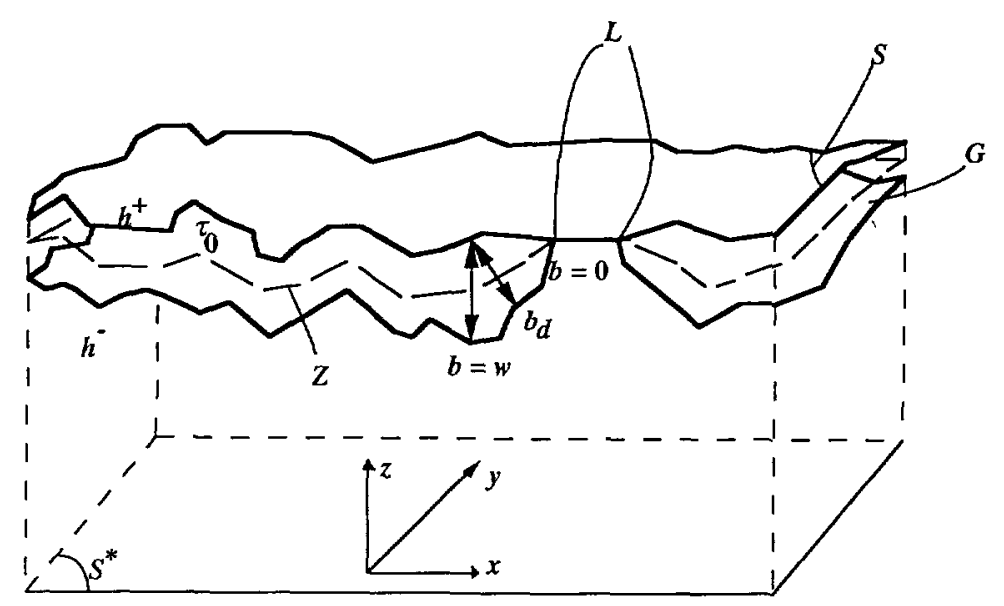

Fig. 1. - Conventions and notation for the fracture geometry. Only a unit cell is displayed.

For the so called Gaussian fractures, all the quantities $h^{+}, h^{-}, Z$ and $w$ were described by the quadratic covariance function

$$
C_{F}(r)=\sigma_{F}^{2} \exp \left(-\left(\frac{r}{l}\right)^{2}\right)
$$

with the same value $l$ of the correlation length. Some attention will also be paid to structures with self-affine correlations $C_{h^{ \pm}}(r)$ which are described at the end of this section.

In practice, spatially periodic fractures are built by juxtaposing identical elements of size $L \times L$, with $L \gg l$. The same approach was used for three-dimensional porous media [8].

The aperture $b$ of a fracture is the difference between $h^{+}$and $h^{-}$when it is non negative

$$
b= \begin{cases}w, & w \geq 0 \\ 0, & w<0\end{cases}
$$

$b$ may be described by its mean aperture $\langle b\rangle$ and its variance $\sigma_{b}^{2}$, which are generally not equal to the mean separation $b_{\mathrm{m}}=\langle w\rangle$ and to $\sigma_{w}^{2}$, respectively. When $w$ is negative, the surfaces are considered to be in contact and one has $h^{+}=h^{-}=Z$.

When the two surfaces of the fracture are very smooth, $b$ is the natural aperture to choose. When this is not the case anymore, $b$ has to be replaced by a better estimate of the local distance between the surfaces, which is inherent in the derivation of the Reynolds equation. Such an aperture can be defined in various ways. One of them consists of the determination of the diameter $b_{\mathrm{d}}$ of the largest sphere able to fit into the fracture at a given node. This method is derived from [9] for the topological characterization of porous media. Let $d_{s}(x)$ denote the distance between the point $x$ which is located in the void space, to the closest solid surface. $b_{\mathrm{d}}(x, y)$ may be formally defined by

$$
b_{\mathrm{d}}(x, y)=2 \max _{z \in\left[h^{-}, h^{+}\right]} d_{\mathrm{s}}(x, y, z)
$$

Instead of $\sigma_{Z}$, the standard deviation $\sigma_{h}$ is used as a characteristic length because it may be directly estimated from measurements $\left(\sigma_{h}^{2}=\sigma_{Z}^{2}+\sigma_{W}^{2} / 4\right)$. The correlation between the two surfaces is characterized by the correlation parameter $\theta=1-\sigma_{W}^{2} / 2 \sigma_{h}^{2}$, which is 0 for uncorrelated surfaces and 1 for fully correlated surfaces All the possible situations can be 
described by the three parameters $b_{m} / \sigma_{h}, l / \sigma_{h}$, and $\theta$; this is equivalent to consider $\sigma_{h}$ as the length unit.

To generate the random fields $Z$ and $w$, the method of Fourier transforms is used (see [8] for details). Gaussian spatially correlated periodic fields were generated on a numerical grid $\left(x_{l}, y_{m}\right)$ with $\Delta x=\Delta y=a$ and $l, m=1, \ldots, N_{c}$ where $N_{c}$ is the number of nodes in each direction. Some examples of vertical sections of generated fractures for different values of $\theta$ are presented in Figure 2. The influence of $\theta$ is clearly seen.

The useful range of parameters can be deduced from an analysis of the experimental data. If the quadratic covariance function (4) is used for $h$, the value $l / \sigma_{h}$ may be found from

$$
\frac{\mathrm{d}^{2} C_{h}}{\mathrm{~d} r^{2}}(r=0)=-\frac{2 \sigma_{h}^{2}}{l^{2}}
$$

This derivative is equal to $-m_{2}$, which is the second moment of the power spectrum of heights. Estimations of $m_{2}$ were presented in [10] for quartzite and granite; $m_{2}$ varied from 0.1 to 0.3 for fractures of different origins. Gentier [11] presented data for $\left\langle(\mathrm{d} h / \mathrm{d} x)^{2}\right\rangle$, which is a statistical estimation of $-C_{h}^{\prime \prime}(0)$, and found that it lies between 0.044 and 0.073 . It is easy to find from (6) that $l / \sigma_{h}$ varies between 2 and 7 .

It is difficult to obtain direct estimates of $b_{\mathrm{m}} / \sigma_{h}$, because $b_{\mathrm{m}}$ is a parameter of the model, while in practice $\langle b\rangle$ is measured. But for a Gaussian distribution (3) of $w$, the value of $b_{\mathrm{m}} / \sigma_{h}$ may be estimated if $\langle b\rangle, \sigma_{h}$ or $\sigma_{b}$ are given. Gentier [11] presented measured values of $\langle b\rangle, \sigma_{h}$ and $\sigma_{b}$. Using these data, $b_{\mathrm{m}} / \sigma_{h}$ was found to vary between 0.2 and 0.8 . Brown
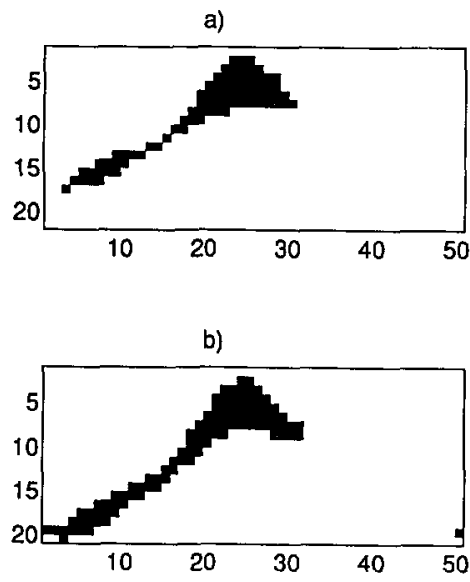

c)

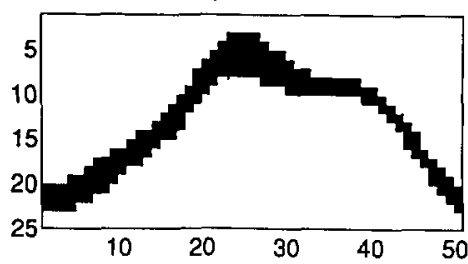

Fig. 2. - Simulated fracture profiles. $l / \sigma_{h}=2$ and $b_{\mathrm{m}} / \sigma_{h}=1$. Data are for: $\theta=0.0$ (a), 0.5 (b) and 0.9 (c). 
et al. [12] measured $\langle b\rangle=0.06-0.4 \mathrm{~mm}, \sigma_{h}=0.23 \mathrm{~mm}$ and $\sigma_{b}=0.078 \mathrm{~mm}$; this implies $0<b_{\mathrm{m}} / \sigma_{h}<2.6$.

To encompass all of these values, $l / \sigma_{h}$ was varied from 1 to $7, b_{\mathrm{m}} / \sigma_{h}$ from 0.5 to 5 and $\theta$ from 0 to 1 . Values of $b_{\mathrm{m}} / \sigma_{h}$ less than 0.5 were not analysed because the fracture space is close to the percolation threshold, and the results of modelling require a refined finite-scale analysis.

Let us now address self-affine fractures since there exist many experimental observations where the self-affine character of rock surfaces is demonstrated [13]. Self-affine surfaces have features of a broad range of characteristic length scales and can be described by the covariance

$$
C_{h}(r) \approx \sigma_{h}^{2}\left(1-\left(\frac{r}{\lambda}\right)^{2 \zeta}\right)
$$

where $\lambda$ is a characteristic length and $\zeta$ is the roughness exponent [14]. For various materials, the exponent $\zeta$ was found to be $0.87 \pm 0.07$ [15].

The spectral density $G(k)$ is the Fourier transform of this covariance $C_{h}(r)$

$$
G(k)=\frac{1}{4 \pi^{2}} \iint C_{h}(r) \mathrm{e}^{2 k r} \mathrm{~d}^{2} r \approx k^{-s}
$$

where $k$ is the wave number. The exponent $s$ is given by

$$
s=2 \zeta+2
$$

$s$ was found to vary between 3 and 4 for various rock surfaces [13]; this is in agreement with the previous estimation of [15].
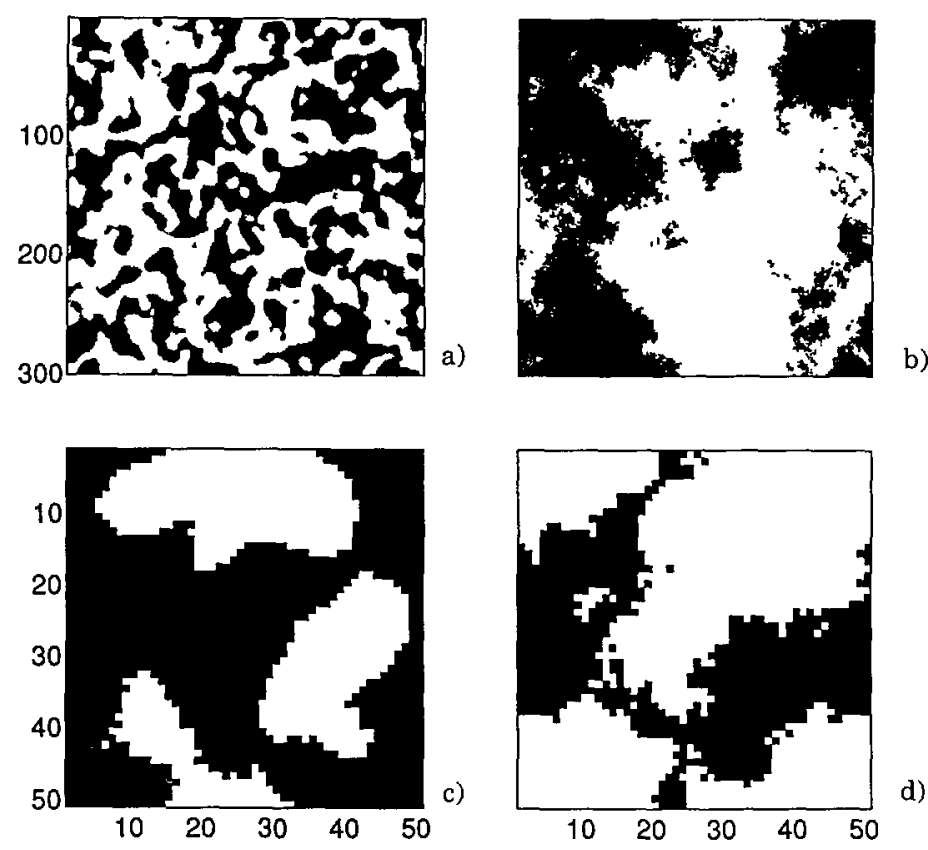

Fig. 3. - Distribution of contacts (white) between two generated surfaces with $b_{\mathrm{m}}=0$. Data are for $L / \sigma_{h}=66$ (a-b), 11 (c-d), (a) and (c) correspond to Gaussian fractures with $l / \sigma_{h}=2$; (b) and (d) correspond to self-affine fractures with $\zeta=08$. 
Since the method of Fourier transforms directly uses the Fourier transform of the covariance function (4a), the spectral density $G(k)$ can be used for the generation of fracture surfaces. Figure 3 shows distributions of contacts between two random surfaces which are generated by using the Gaussian covariance (4b) and the self-affine spectral density (8). The difference between the visual aspects of these two examples is striking.

It should be noticed that there are two cut-offs in the generation of self-affine fractures. The lower cut-off corresponds to the size of the elementary square $a$; the larger cut-off corresponds to the half-size of the unit cell itself. As for Gaussian fractures, spatially periodic conditions were used at the boundaries of the unit cell.

\section{Stokes Equations}

It is assumed in this paper that the velocities and characteristic dimensions are so small that inertial effects are negligible. Under these conditions which can be summarized by saying that the Reynolds number is vanishingly small, the Navier-Stokes equations can be replaced by the Stokes equations; this assumption is also valid when step-wise variations in aperture arise (cf. Fig. 2).

The analysis of Stokes flow is very similar to that made for three-dimensional porous media (cf. [8]). Consider an infinite fracture made of identical unit cells of size $L \times L$ in the $(x, y)$ plane. The low Reynolds number flow of an incompressible Newtonian fluid through a fracture is governed by the usual 3D Stokes equations

$$
\nabla p=\mu \nabla^{2} \mathbf{v}, \quad \nabla \cdot \mathbf{v}=0
$$

where $\mathbf{v}, p$ and $\mu$ are the velocity, pressure and viscosity of the fluid, respectively. In general, $v$ satisfies the conditions

$$
\begin{gathered}
\mathbf{v}=0, \quad \text { on the total surface } S \\
\text { of the fracture }
\end{gathered}
$$

and

$$
\begin{gathered}
\mathbf{v} \text { is spatially periodic with } \\
\text { period } L \text { in the plane } \\
\text { of the fracture }
\end{gathered}
$$

This system of equations and conditions applies locally at each point $R$ of the interstitial fluid. In addition, it is assumed that the macroscopic pressure gradient $\overline{\nabla p}$ is specified,

$$
\overline{\nabla p}=\frac{1}{\tau_{0}} \int_{\partial \tau_{0}} p \mathrm{~d} s=\text { a prescribed constant vector }
$$

where $\tau_{0}$ is the volume of the fracture; $\tau_{0}$ is bounded by the two solid surfaces $S$ and the vertical boundary of the unit cell $G$, so that $\partial \tau_{0}=S \cup G$ (see Fig. 1). The mean flow rate per unit fracture width $\overline{\mathbf{Q}_{S}}$ may be defined as

$$
\overline{\mathbf{Q}_{S}}=\frac{1}{S^{*}} \int_{\tau_{0}} \mathbf{v} \cdot d^{3} \mathbf{r}
$$


where $S^{*}$ is the area of the $(x, y)$-projection of $S . \overline{\mathbf{Q}_{S}}$ is linearly related to the pressure gradient $\nabla p$ by the Stokes permeability tensor $\mathbf{B}_{\mathrm{s}}$

$$
\overline{\mathbf{Q}_{S}}=-\frac{1}{\mu} \mathbf{B}_{\mathbf{s}} \cdot \overline{\nabla p}
$$

$\mathbf{B}_{\mathrm{s}}$ is a positive definite symmetric tensor which only depends on the geometry of the system; for isotropic fracture surfaces, the average tensor $\mathbf{B}_{\mathrm{s}}$ may be simplified as $\mathbf{B}_{\mathrm{s}}=B_{\mathrm{s}} \cdot \mathbf{I}$, where $\mathbf{I}$ is the unit tensor. The value $b_{\mathrm{S}}=\left(12 B_{\mathrm{s}}\right)^{1 / 3}$ is defined as the hydraulic aperture of a fracture as estimated from the Stokes equations.

Further details on some technical points such as the spatially periodic boundary conditions can be found in [8].

The numerical method which is used here is the so-called artificial compressibility method and is an improved version of that used in [16]. The problem is replaced by an unsteady compressible one which is assumed to converge towards the steady incompressible situation of interest. The equations were solved successively along each direction by using an alternatingdirection-implicit scheme.

Convergence was reached when the flow rate was found to be the same within $1 \%$ across various sections of the medium. It sometimes took more than 104 iterations to obtain the solution. This number critically depends upon the geometry of the sample. The time step is limited by the occurrence of numerical instabilities and could not be increased during a given calculation to accelerate convergence.

A $50 \times 50$ mesh in the $(x, y)$-plane was used for most realizations. The number of nodes in the $z$-direction was varied from 25 to 40 and depended upon the distance $b_{\mathrm{m}} \cdot \mathbf{B}_{\mathrm{s}}$ varied of about $8 \%$ when $N_{\mathrm{c}}$ varied from 40 to 80 . The step size $a$ was usually equal to $0.2 \sigma_{h}$ and was increased to $0.45 \sigma_{h}$ for $b_{\mathrm{m}} / \sigma_{h}=10$. This discretisation is supposed to be small enough for a good representation of the vertical variations of the surface profiles. Since the correlation distance verifies $l / \sigma_{h} \geq 1$, the horizontal surface structures are also well represented (see Fig. 2).

It should be noted that the quadratic covariance function (4b) provides a random field which has no significant small scale features for lengths much smaller than $l$ (Fig. 3a). The exponential decrease of correlations between heights at different points implies that the probability of appearance of large structures with a size $x \gg l$ is also negligible. This is not true for self-affine surfaces where structures of different length scales can appear with a comparable probability (Fig. 3b). The numerical method used here can be applied to flow in fractures with self-affine surfaces, but only if a limited range of characteristic length scales is taken into account, namely between the lower and upper cut-offs $a$ and $L / 2$ which were mentioned before.

\section{Reynolds Equation}

It is usually assumed that for very small Reynolds numbers and for slowly varying fractures, i.e. in the limit where $b_{\mathrm{m}} / \sigma_{h}$ and $l / \sigma_{h}$ are large; the Navier-Stokes equations can be reduced locally to the two-dimensional Reynolds equation [3]

$$
\nabla \cdot\left(b^{3} \nabla p\right)=0
$$

Both definitions $b$ and $b_{\mathrm{d}}\left(5 \mathrm{a}\right.$ and $\mathrm{b}$ ) can be used in this equation. When $b_{\mathrm{d}}$ is used, the resulting quantities are indexed by the subscript $d$.

Equation (15a) has to be supplemented by the no-flux boundary condition at the solid boundary $L$ of the contact region (cf. also [17]). Let $\mathbf{n}$ be the normal in the $x y$-plane to the curve $L$; this condition reads as 


$$
\mathbf{n} \cdot \nabla p=0 \text { on } L
$$

The flow rate per unit fracture width $\mathbf{Q}$ is given by

$$
\mathbf{Q}=-\frac{b^{3}}{12 \mu} \nabla p
$$

As previously, an infinite fracture made of identical unit cells of size $L \times L$ in the $(x, y)$ plane is considered. A complete analogy between the Reynolds equation and a two-dimensional diffusion problem exists (cf. [8]). It is supposed that $\mathbf{Q}$ is spatially periodic and $\overline{\nabla \mathbf{p}}$ is the same constant vector as in (12). In the same way, the mean flux $\overline{\mathbf{Q}_{\mathrm{R}}}$ is defined as

$$
\overline{\mathbf{Q}_{\mathbf{R}}}=\frac{1}{S^{*}} \int_{S^{*}} \mathbf{Q} \mathrm{d} s
$$

The Reynolds permeability tensor $\mathbf{B}_{\mathrm{R}}$ is readily defined by

$$
\overline{\mathbf{Q}_{\mathbf{R}}}=-\frac{1}{\mu} \mathbf{B}_{\mathbf{R}} \cdot \overline{\nabla \mathbf{p}}
$$

The hydraulic aperture is calculated from $b_{\mathrm{R}}=\left(12 B_{\mathrm{R}}\right)^{1 / 3}$, where $B_{\mathrm{R}}$ is found after the averaging of $\mathbf{B}_{\mathrm{R}}$ over a large number of statistical realizations; again for statistically isotropic fractures, $\mathbf{B}_{\mathrm{R}}=B_{\mathrm{R}} \cdot \mathbf{I}$. The analogous quantities $B_{\mathrm{Rd}}$ and $b_{\mathrm{Rd}}$ will be often used in the following.

To solve the elliptic equation (15) together with the spatially periodic boundary conditions, the numerical "box integration method" is used (see $[8,18,19]$ ). The method was originally developed to solve usual diffusion equation with variable coefficients. The medium can be considered as the union of a set of elementary squares, over which $b^{3}$ is constant. The pressure field is defined at nodes, which are the corners of these elementary squares.

The elliptic equation (15a) is integrated over cells centered around each node. Truncated Taylor series are used for this integration. The discretized linear equation for the pressure field is obtained and is completed by overall boundary conditions. The set of equations for $p$ was replaced by a time dependent one which is assumed to converge towards the steady solution. A time explicit scheme was used and in most cases an accurate solution is obtained in less than one thousand iterations.

\section{Results and Discussion}

The purpose of this study was to calculate solutions of the 2D Reynolds equation and of the 3D Stokes equations for the same fracture geometry and to compare them. The major portion of this section will address Gaussian fractures. Figure 4 shows generated aperture distribution and calculated flux fields for fractures with non-correlated surfaces $(\theta=0)$ for three of the four possibilities corresponding to the two values 1 and 3 of the ratios $1 / \sigma_{h}$ and $b_{m} / \sigma_{h}$. The simulations were run on a $50 \times 50 \times 35$ mesh with a step size $a=0.22 \sigma_{h}$. The unit for the aperture fields $b$ and $b_{\mathrm{d}}$ is chosen to be $\sigma_{h}$, in agreement with the previous conventions. The unit for the flow rate per unit fracture width $Q$ is chosen to be $\sigma_{h}^{3} \overline{\nabla p} / \mu$. Due to large differences between the maximal values of $b$ and $Q$ for various geometrical parameters of the generated fractures, different levels of shading are used; the choice of $b_{\mathrm{m}}^{3} \cdot \bar{\nabla} p / \mu$. would certainly reduce these differences.

It is interesting to compare the first two rows. The overall shape of the aperture distributions is conserved, but with large quantitative differences. 


$\begin{array}{ccc}\text { Column }(1) & \text { Column }(2) & \text { Column }(3) \\ l / \sigma_{h}=1, b_{m} / \sigma_{h}=1 & l / \sigma_{h}=1, b_{m} / \sigma_{h}=3 & l / \sigma_{h}=3, b_{m} / \sigma_{h}=3 \\ \Delta b=12, \Delta b_{d}=06, & \Delta b=12, \Delta b_{d}=06, & \Delta b=12, \Delta b_{d}=06, \\ \Delta Q_{l, s}=02, \Delta Q_{R}=0.1, & \Delta Q_{l, s}=02, \Delta Q_{R}=1, & \Delta Q_{l} s=02, \Delta Q_{R}=1, \\ \Delta Q_{R d}=001 & \Delta Q_{R d}=02 & \Delta Q_{R d}=02\end{array}$
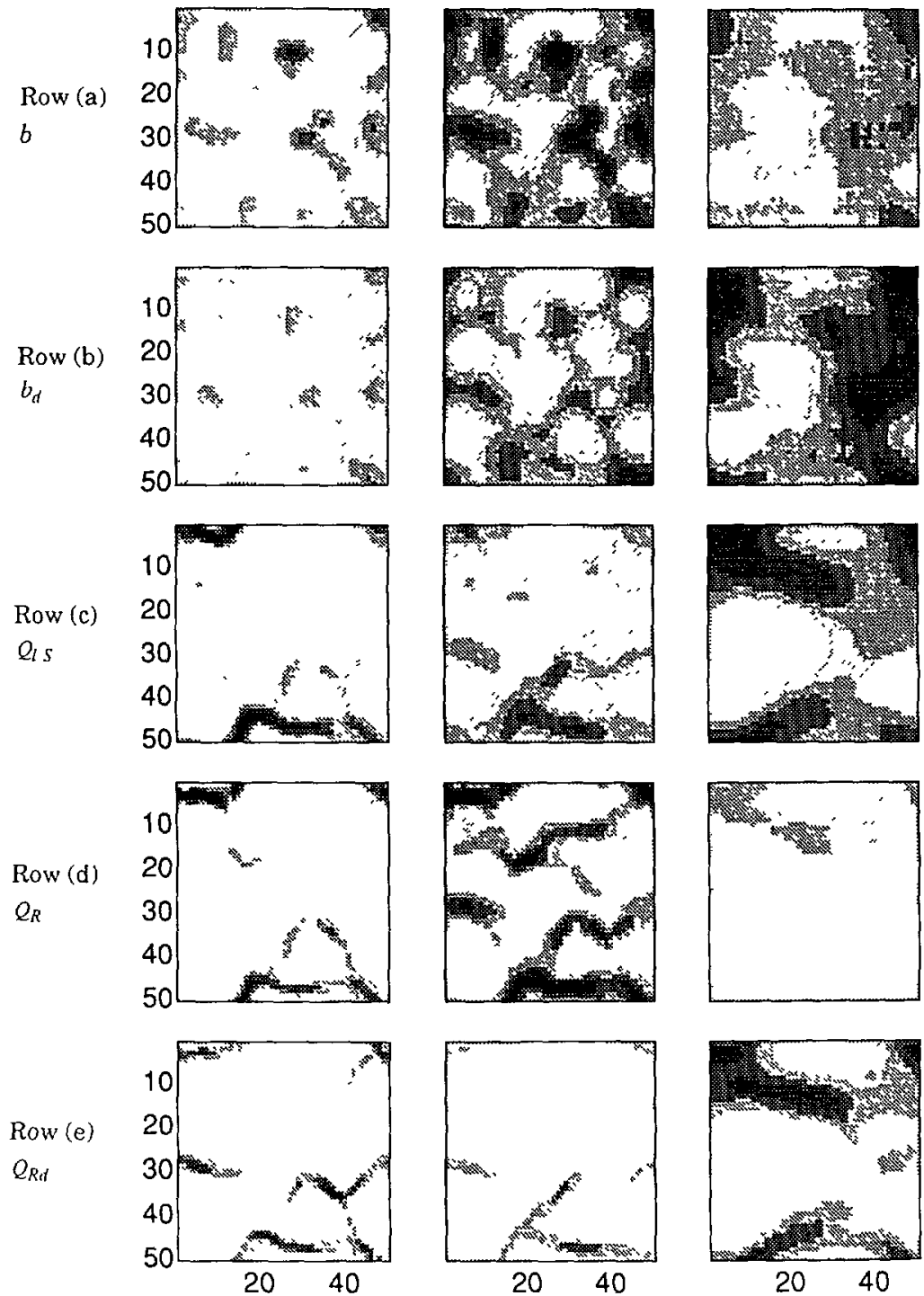

Fig. 4. - Simulated aperture and computed flux distributions over the fracture plane for noncorrelated Gaussian fractures $(\theta=0)$. Six levels of shadings are distinguished from zero (white) to the largest value (dark). The shading steps for aperture are equal to $\Delta b$; for fluxes, they are equal to $\Delta Q$. The three columns correspond to three combinations of the geometrical parameters $l / \sigma_{h}$ and $b_{\mathrm{m}} / \sigma_{h}$. The first two rows correspond to $b$ and $b_{\mathrm{d}}$ with steps $\Delta b=1.2$ and $\Delta b_{\mathrm{d}}=0.6$. The last three rows correspond to the three fluxes $Q_{l, \mathrm{~s}}$ (Stokes equations), $Q_{\mathrm{R}}$ (Reynolds equation with aperture $b$ ) and $Q_{\mathrm{Rd}}$ (Reynolds equation with aperture $b_{\mathrm{d}}$ ) 
It is easy to find that with decreasing $b_{\mathrm{m}}$, the flow becomes more heterogeneous and fluid passes through several preferential flow paths (Figs. 4.c.1 and 2). This is the so-called channeling effect of fluid flow in fractures, which was observed experimentally [20] and described in numerical simulations $[2,21]$. Flow channeling appears for both Stokes and Reynolds flows (Figs. 4.1.c, $\mathrm{d}$ and e). For large values of the correlation length $l / \sigma_{h}$, flow channels become larger (Figs. 4.c.2 and 3 ) because the aperture $b$ varies less with the distance than for low $l$ (Figs. 4.a.2 and 3).

The general features of the flow distributions obtained by the Reynolds model with $b$ and $b_{\mathrm{d}}$ are roughly the same, especially for small $b_{\mathrm{m}} / \sigma_{h}$ (Figs. $4 \mathrm{~d} .1$ and e.1). This is due to the fact that the distribution of $b_{\mathrm{d}}$ approximately follows the distribution of $b$ (Figs. $4 \mathrm{a}$ and $\mathrm{b}$ ). The preferential flow paths are expected to be almost the same, because they are determined by the channels with the largest aperture.

In order to quantitatively compare the flow distributions between the Stokes and Reynolds models, the two cross-covariances were calculated

$$
\begin{gathered}
C_{\mathrm{fl}}=\int_{S^{*}}(Q-\bar{Q}) \cdot\left(Q_{l, \mathrm{~S}}-\overline{Q_{l, \mathrm{~S}}}\right) \mathrm{d} s /\left[\int_{S^{*}}(Q-\bar{Q})^{2} \mathrm{~d} s \cdot \int_{S^{*}}\left(Q_{l, \mathrm{~S}}-\overline{Q_{l, \mathrm{~S}}}\right)^{2} \mathrm{~d} s\right]^{1 / 2} \\
\overline{\mathbf{Q}_{\imath}}=\frac{1}{S^{*}} \int \mathbf{Q}_{\imath} \mathrm{d} s
\end{gathered}
$$

The local flux $\mathbf{Q}_{l, \mathrm{~S}}$ in the Stokes approximation can be defined as

$$
\mathbf{Q}_{l, S}=\int_{-h}^{h^{+}} \mathbf{v}(\mathbf{x}) \mathrm{d} z
$$

The subscript $l$ was added in order to avoid any confusion. The local flux $\mathbf{Q}$ in the Reynolds approximation is given by (16) where either $b$ or $b_{\mathrm{d}}$ is used.

$C_{\mathrm{f}}$ and $C_{\mathrm{fld}}$ are given in Table I for various values of $l / \sigma_{h}$ and $b_{\mathrm{m}} / \sigma_{h}$. These data correspond to a single fracture for each value of $l / \sigma_{h}$ and $\theta$. Note that for high $b_{\mathrm{m}} / \sigma_{h}$, the flow distributions between the Stokes and Reynolds models are more different than they are for low $b_{\mathrm{m}} / \sigma_{h}$. This trend can be observed for both Reynolds models and various values of $l / \sigma_{h}$ and $\theta$; this is not true anymore for small $b_{\mathrm{m}} / \sigma_{h}$ (see Tab I). This is surprising because the influence of surface roughness should decrease and because the flow distribution should approach the Poiseuille parabolic distribution, when $b_{\mathrm{m}}$ increases. For most realizations $C_{\mathrm{fld}}$ is found to be larger than $C_{\mathrm{fl}} \cdot C_{\mathrm{fld}}$ is about 0.9 when $b_{\mathrm{m}}>l$, whereas it drops rapidly when the mean separation $b_{\mathrm{m}}$ becomes smaller than the correlation length $l$; the same statement holds for highly correlated surfaces $(\theta=0.9)$.

Figure 5 shows the same fields for highly-correlated surfaces $(\theta=0.9)$. It is obvious from the figures that a small value $b_{\mathrm{m}}$ of favours flow channeling in both models (Fig. 5,c-d, 1 and 2), but here flow is distributed over a larger number of flow paths than for $\theta=0$. The difference in flow patterns obtained with Reynolds and Stokes equations increases when $b_{\mathrm{m}}$ increases for $\theta=0.9$ as can be seen in Table $\mathrm{I}$.

The overall permeabilities were analysed by means of the ratios

$$
\frac{B_{\mathrm{h}}}{B_{\mathrm{p} 1}}=\left(\frac{b_{\mathrm{h}}}{<b>}\right)^{3} ; \quad \frac{B_{\mathrm{h}}}{B_{\mathrm{p}_{2}}}=\left(\frac{b_{\mathrm{h}}}{b_{\mathrm{m}}}\right)^{3}, \quad h=S, R, R_{\mathrm{d}}
$$

where $B_{\mathrm{p}_{1}}=<b>^{3} / 12$ and $B_{\mathrm{p}_{2}}=b_{\mathrm{m}}^{3} / 12$ are the effective permeabilities of a plane channel of heights $\langle b\rangle$ and $b_{\mathrm{m}}$, respectively. 

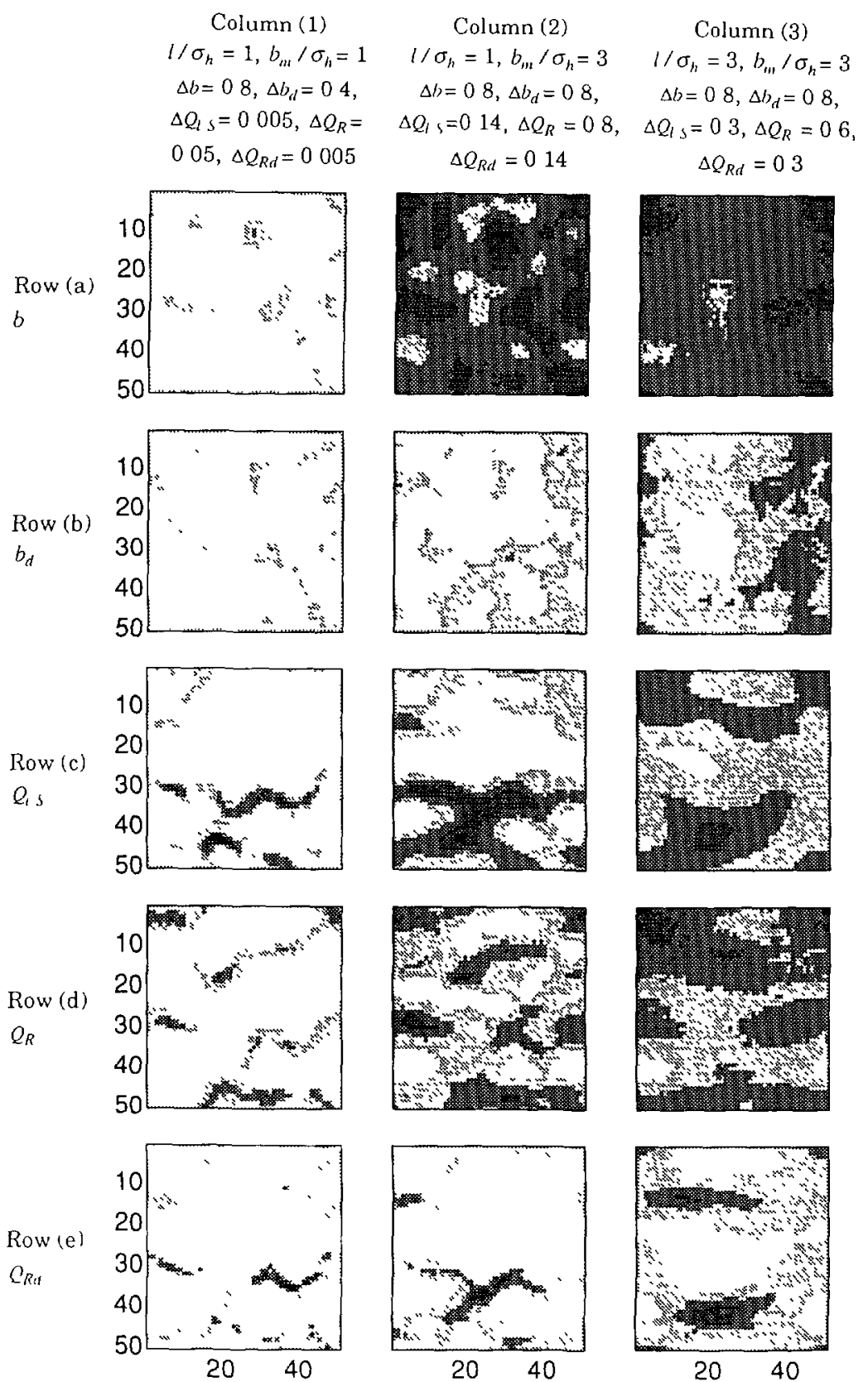

Fig. 5. - Simulated aperture and computed flux distributions over the fracture plane for correlated Gaussian fractures $(\theta=0.9)$. Six levels of shadings are distıngushed from zero (white) to the largest value (dark) The shading steps for aperture are equal to $\Delta b$, for fluxes, they are equal to $\Delta Q$. The three columns correspond to three combinations of the geometrical parameters $l / \sigma_{h}$ and $b_{\mathrm{m}} / \sigma_{h}$. The first two rows correspond to $b$ and $b_{\mathrm{d}}$. The three last rows correspond to the three fluxes $Q_{l, \mathrm{~S}}$ (Stokes equations), $Q_{\mathrm{R}}$ (Reynolds equation with aperture $b$ ) and $Q_{\mathrm{Rd}}$ (Reynolds equation with aperture $b_{\mathrm{d}}$ ). 
Table I. - The cross-covariances $C_{\mathrm{fl}}$ and $C_{\mathrm{fld}}$ of local fluxes. Gaussian fractures.

\begin{tabular}{|c|c|c|}
\hline$b_{m} / \sigma_{h}$ & $C_{f l}$ & $C_{\text {fld }}$ \\
\hline \multicolumn{3}{|c|}{$\theta=0, l / \sigma_{h}=1$} \\
\hline 10.0 & 0.57 & 0.84 \\
6.0 & 0.68 & 0.91 \\
3.0 & 0.81 & 0.92 \\
2.5 & 0.82 & 0.91 \\
2.0 & 0.83 & 0.92 \\
1.5 & 0.84 & 0.89 \\
1.0 & 0.83 & 0.73 \\
0.5 & 0.79 & 0.26 \\
\hline \multicolumn{3}{|c|}{$\theta=0, l / \sigma_{h}=3$} \\
\hline 10.0 & 0.90 & 0.93 \\
6.0 & 0.91 & 0.94 \\
3.0 & 0.95 & 0.95 \\
2.5 & 0.96 & 0.95 \\
2.0 & 0.96 & 0.50 \\
1.5 & 0.96 & 0.83 \\
1.0 & 0.84 & 0.34 \\
0.5 & - & - \\
\hline \multicolumn{3}{|c|}{} \\
\hline 10.0 & $\theta=0.9, l / \sigma_{h}=1$ & 0.84 \\
6.0 & 0.21 & 0.86 \\
3.0 & 0.13 & 0.88 \\
2.5 & 0.43 & 0.88 \\
2.0 & 0.43 & 0.87 \\
1.5 & 0.47 & 0.87 \\
1.0 & 0.52 & 0.78 \\
0.5 & 0.56 & 0.24 \\
\hline
\end{tabular}

For the same values of $b_{\mathrm{m}}, l$ and $\theta$, several realizations of $Z$ and $w$ were used to obtain statistically representative results. All simulations were performed on a $50 \times 50 \times N_{z}$ mesh, where $N_{z}$ was varied from 25 for $b_{\mathrm{m}} / \sigma_{h} \leq 2$ to 35 for $b_{\mathrm{m}} / \sigma_{h}=5$. Comparison between simulations performed on this $50 \times 50 \times N_{z}$ mesh and simulations performed on a $80 \times 80 \times 50$ mesh, for $b_{\mathrm{m}} / \sigma_{h}=1, l / \sigma_{h}=1$, showed that $b_{\mathrm{R}}^{3}$ and $b_{\mathrm{S}}^{3}$ varied by only $8 \%$. This is much smaller than the difference between Reynolds and Stokes models.

Each realization of pair of fracture surfaces has been combined for various values of $b_{\mathrm{m}} / \sigma_{h}$. Eight values of $b_{\mathrm{m}} / \sigma_{h}$ ranging from 0.5 to 10 were used, while $l / \sigma_{h}$ varied from 1 to 3 . Ratios $B_{h} / B_{\mathrm{p}_{1}}$ averaged over 5 realizations of pairs of fracture surfaces are presented in Figure 6. One may conclude that if $b$ is used as the local aperture in the Reynolds equation (15), the resulting mean flow rate is significantly larger than the one derived from the Stokes equation. The simulation with $b_{\mathrm{d}}$ yields a better approximation of $B_{\mathrm{S}}$, at least for $b_{\mathrm{m}} / \sigma_{h} \geq 2$. Within the interval $2 \leq b_{\mathrm{m}} / \sigma_{h} \leq 10$, the trends of $B_{\mathrm{S}}$ and $B_{\mathrm{Rd}}$ are similar.

For $b_{\mathrm{m}} / \sigma_{h}<2, B_{\mathrm{Rd}}$ undergoes large fluctuations with $b_{\mathrm{m}} / \sigma_{h}$. This may be due to the fact that $b_{\mathrm{m}} / \sigma_{h}$ becomes comparable to the mesh size; hence, numerical results are not reliable anymore.

The calculated values of the ratios $B_{\mathrm{R}} / B_{\mathrm{p}_{1}}$ are in a good qualitative agreement with the 


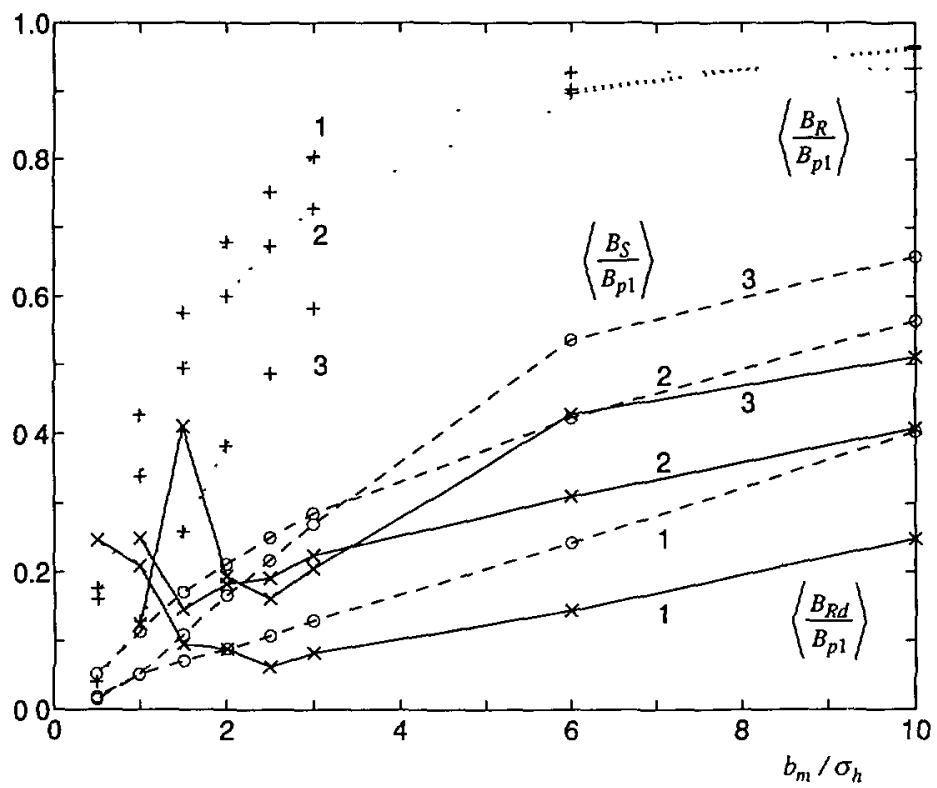

Fig. 6. - Dimensionless permeabilities $\left\langle B_{\mathrm{Rd}} / B_{p_{1}}\right\rangle$ (solid lines), $\left\langle B_{\mathrm{S}} / B_{p_{1}}\right\rangle$ (dashed lines), and $\left\langle B_{\mathrm{R}} / B_{p_{1}}\right\rangle$ (dotted lines) as functions of the mean separation $b_{\mathrm{m}} / \sigma_{h}$ for $\theta=0$ and various $l / \sigma_{h}(1,2$ and 3 ). Gaussian fractures.

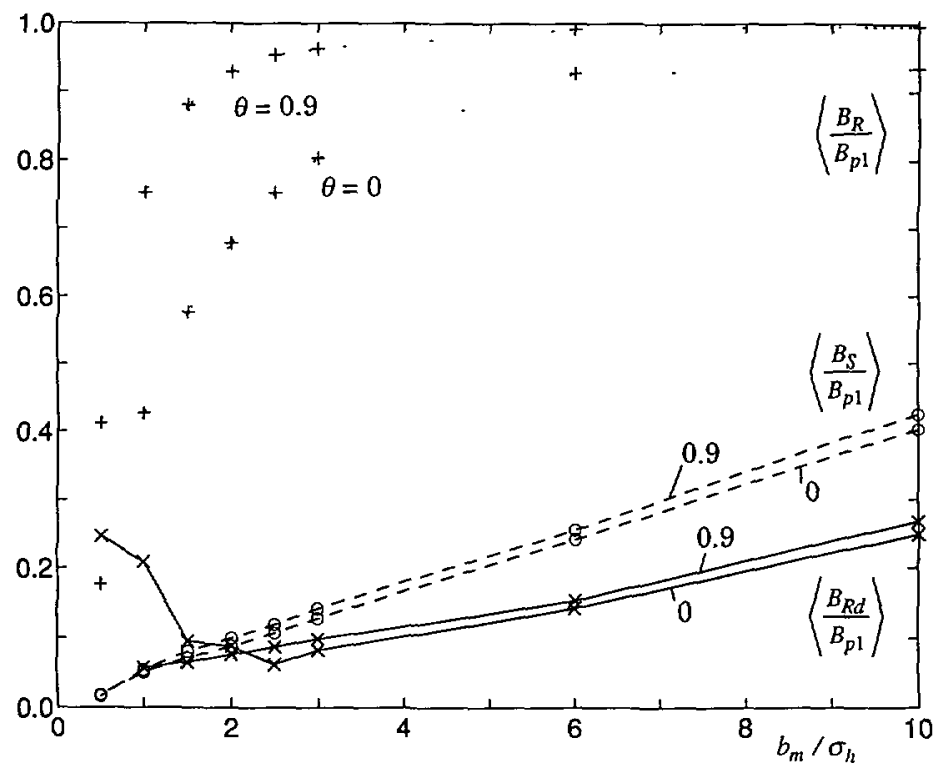

Fig 7. - Dimensionless permeabilities $\left\langle B_{\mathrm{Rd}} / B_{p_{1}}\right\rangle$ (solid lines), $\left\langle B_{\mathrm{S}} / B_{p_{1}}\right\rangle$ (dashed lines), and $\left\langle B_{\mathrm{R}} / B_{p_{1}}\right\rangle$ (dotted lines) as functions of the mean separation $b_{\mathrm{m}} / \sigma_{h}$ for $l / \sigma_{h}=1$ and various $\theta$ (0 and 0 9) Gaussian fractures. 
numerical results of Brown [2] who used the Reynolds equation (15) with the aperture defined by $(5 \mathrm{a})$. He found that $B_{\mathrm{R}} / B_{\mathrm{p}_{1}}$ is unsensitive to the slope of the power spectrum of surface profiles, which defines the spatial correlation of surface roughness. The results of the present simulations also show that $B_{\mathrm{R}} / B_{\mathrm{p}_{1}}$ does not depend upon $l / \sigma_{h}$ for $b_{\mathrm{m}} / \sigma_{h} \geq 6$ (Fig. 6). This is not true for $B_{\mathrm{S}}$ and $B_{\mathrm{Rd}}$, which decrease with decreasing $l / \sigma_{h}$.

Figure 7 shows $\left\langle B_{h} / B_{\mathrm{p}_{1}}\right\rangle$ for five realizations of fractures with correlated $(\theta=0.9)$ and uncorrelated $(\theta=0)$ surfaces. It is clear that $\theta$ only slightly influences $B_{\mathrm{S}}$ and $B_{\mathrm{Rd}}$ (for $b_{\mathrm{m}} / \sigma_{h} \geq 2$ ), but largely influences $B_{\mathrm{R}}$.

Hydraulic apertures are compared to the mean separation $b_{\mathrm{m}}$ of fracture surfaces in Figure 8 . All the curves show the same trend; $B_{h} / B_{\mathrm{p}_{2}}$ is an increasing function of $b_{\mathrm{m}} / \sigma_{h}$, for $b_{\mathrm{m}} / \sigma_{h} \geq$ $1 \sim 2$; for $b_{\mathrm{m}} / \sigma_{h} \leq 1 \sim 2$, the ratio $B_{h} / B_{p_{2}}$ decreases with $b_{\mathrm{m}} / \sigma_{h}$. It should be noted that the calculated values of the ratios $B_{\mathrm{R}} / B_{p_{2}}$ are also in agreement with the numerical results of [2]. As expected, for large fracture apertures, $b_{\mathrm{R}},\langle b\rangle$ and $b_{\mathrm{m}}$ become equivalent, whereas $b_{\mathrm{Rd}}$ is significantly smaller. This is due to the fact that the distance $b_{\mathrm{d}}$ is very sensitive to all the asperities of the fracture surfaces. As a general rule, $b_{\mathrm{S}}$ lies between $b_{\mathrm{Rd}}$ and $b_{\mathrm{R}}$.

Finally, the variations of $\left\langle B_{\mathrm{R}} / B_{\mathrm{S}}\right\rangle$ and $\left\langle B_{\mathrm{Rd}} / B_{\mathrm{S}}\right\rangle$ with the two parameters $l / \sigma_{h}$ and $b_{\mathrm{m}} / \sigma_{h}$ are shown in Figures 9 and 10, and in Table II. Clearly, the best comparisons are observed for large values of $b_{\mathrm{m}} / \sigma_{h}$ and $l / \sigma_{h}$, i.e. for wide and smooth fractures. It was found that for $l / \sigma_{h} \leq 3, b_{\mathrm{m}} / \sigma_{h} \leq 3$, the ratio $\left\langle B_{\mathrm{R}} / B_{\mathrm{S}}\right\rangle$ is always larger than 2 and depends slightly upon $\theta$. This means that for the range of values $l / \sigma_{h}$ and $b_{\mathrm{m}} / \sigma_{h}$ under consideration, the Reynolds equation with a locally distributed aperture (which is the difference between upper and lower surfaces) gives a total flow rate per unit fracture width more than 2 times larger than the Stokes equations. The difference is maximal for $l / \sigma_{h}=1, b_{\mathrm{m}} / \sigma_{h}=0.5$, where the ratio reaches 9.2

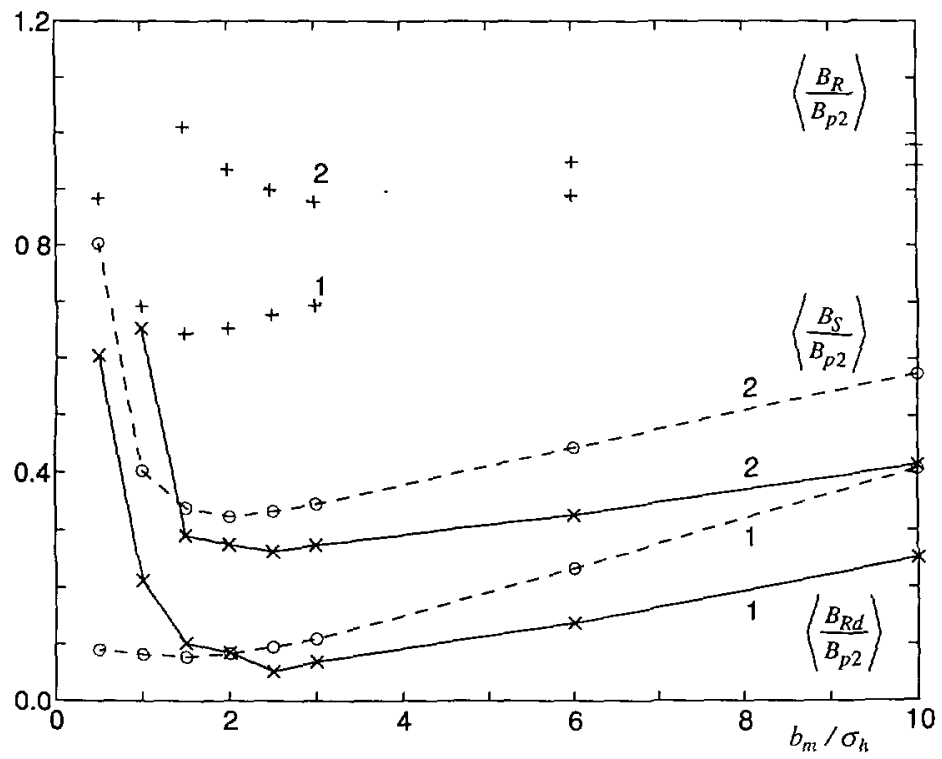

Fig. 8 -Dimensionless permeabilities $\left\langle B_{\mathrm{Rd}} / B_{p_{2}}\right\rangle$ (solıd lines), $\left\langle B_{\mathrm{S}} / B_{p_{2}}\right\rangle$ (dashed lines), and $\left\langle B_{\mathrm{R}} / B_{p_{2}}\right\rangle$ (dotted lines) as functions of the mean separation $b_{\mathrm{m}} / \sigma_{h}$ for $\theta=0$ and various $l / \sigma_{h}(1$ and 2) Gaussian fractures. 


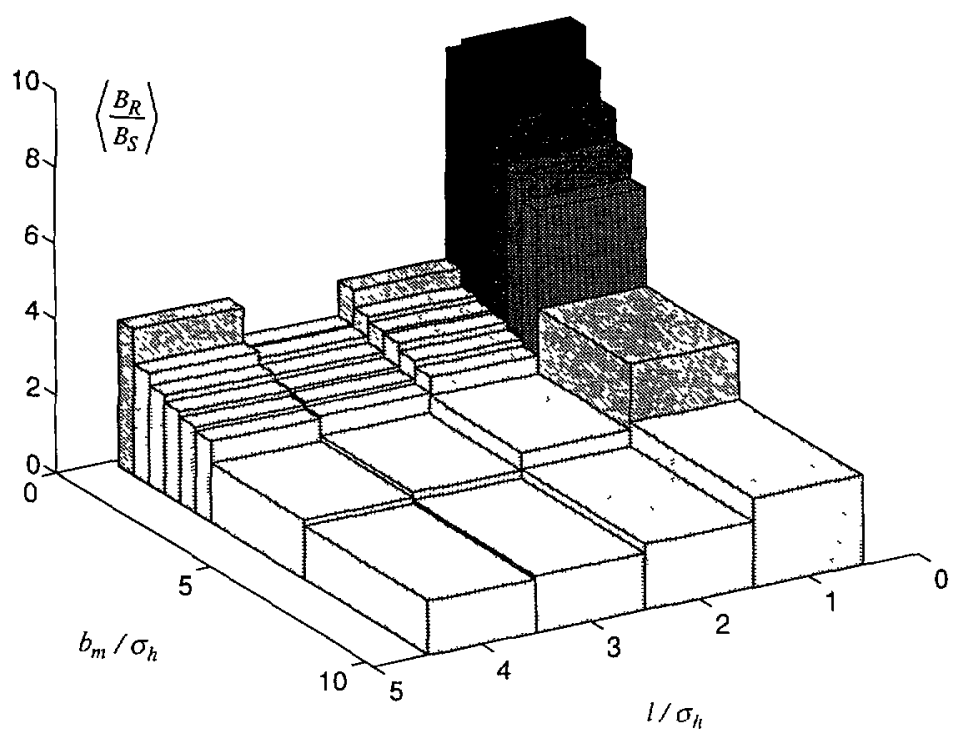

Fig. 9 - The ratio $\left\langle B_{\mathrm{R}} / B_{\mathrm{S}}\right\rangle$ as a function of $l / \sigma_{h}=1$ and $b_{\mathrm{m}} / \sigma_{h}=1$ for Gaussian fractures with non-correlated surfaces $(\theta=0), b$ is used in the Reynolds equation. The block basis corresponds to $\left\langle B_{\mathrm{R}} / B_{\mathrm{S}}\right\rangle=0$.

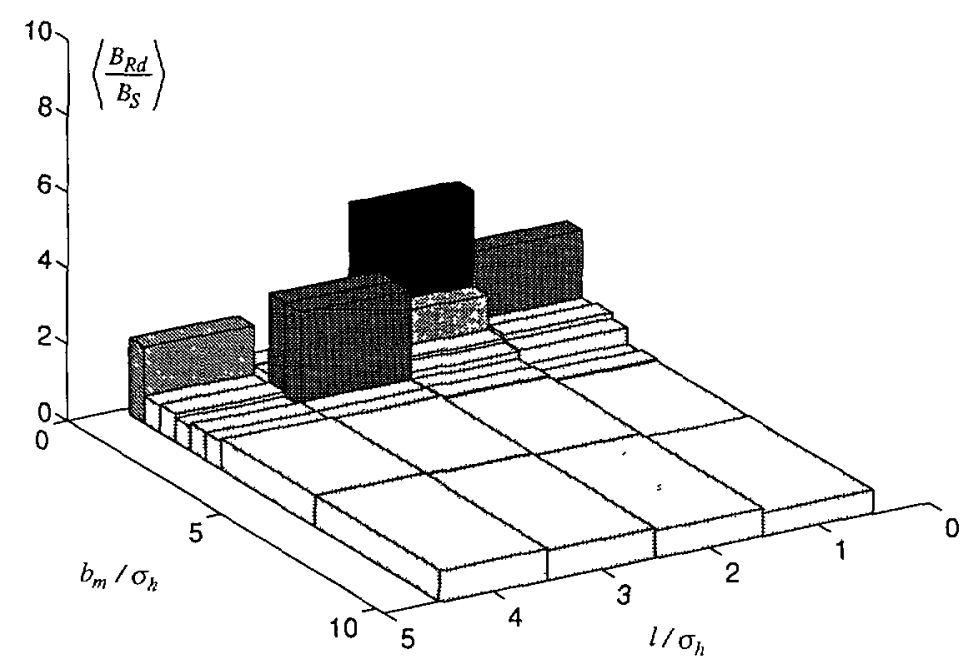

Fig. 10 - The ratio $\left\langle B_{\mathrm{Rd}} / B_{\mathrm{S}}\right\rangle$ as a function of $l / \sigma_{h}$ and $b_{\mathrm{m}} / \sigma_{h}$ for Gaussian fractures with noncorrelated surfaces; $b_{\mathrm{d}}$ is used in the Reynolds equation The block basis corresponds to $\left\langle B_{\mathrm{Rd}} / B_{\mathrm{S}}\right\rangle=0$.

$\theta$ was not found to influence the ratio $<B_{\mathrm{R}} / B_{\mathrm{S}}>$ significantly, but simulations gave unexpected results. For highly correlated surfaces, the ratio $B_{\mathrm{R}} / B_{\mathrm{S}}$ is greater than for uncorrelated surfaces $(\theta=0)$. This is surprising because for $\theta \approx 1$, the surfaces have almost the same form; the fracture aperture varies very slowly and one may expect that under these conditions, flow is close to a Poiseuille flow. Table II shows that the difference between the cases $\theta=0.9$ and $\theta=0$ is smaller than $60 \%$ for $l / \sigma_{h}=1, b_{\mathrm{m}} / \sigma_{h} \geq 1$ and that this difference decreases with $l$. 
Table II. - The ratio $\left\langle B_{\mathrm{R}} / B_{\mathrm{S}}\right\rangle$ as a function of $l / \sigma_{h}, b_{\mathrm{m}} / \sigma_{h}$ and $\theta$. Gaussian fractures.

\begin{tabular}{|c|c|c|c|c|}
\hline$b_{m} / \sigma_{h}$ & \multicolumn{2}{|c|}{$l / \sigma_{h}=1$} & \multicolumn{2}{c|}{$l / \sigma_{h}=3$} \\
\hline & $\theta=0$ & $\theta=0.9$ & $\theta=0$ & $\theta=0.9$ \\
\hline 10.0 & 2.32 & 2.35 & 1.46 & 1.44 \\
6.0 & 3.84 & 3.90 & 1.67 & 1.59 \\
3.0 & 6.44 & 7.18 & 2.20 & 1.93 \\
2.5 & 7.20 & 8.45 & 2.32 & 2.02 \\
2.0 & 8.00 & 9.96 & 2.41 & 2.15 \\
1.5 & 8.83 & 12.03 & 2.53 & 2.34 \\
1.0 & 9.66 & 16.14 & 2.69 & 2.89 \\
0.5 & 9.20 & 26.06 & 2.76 & 7.10 \\
\hline
\end{tabular}

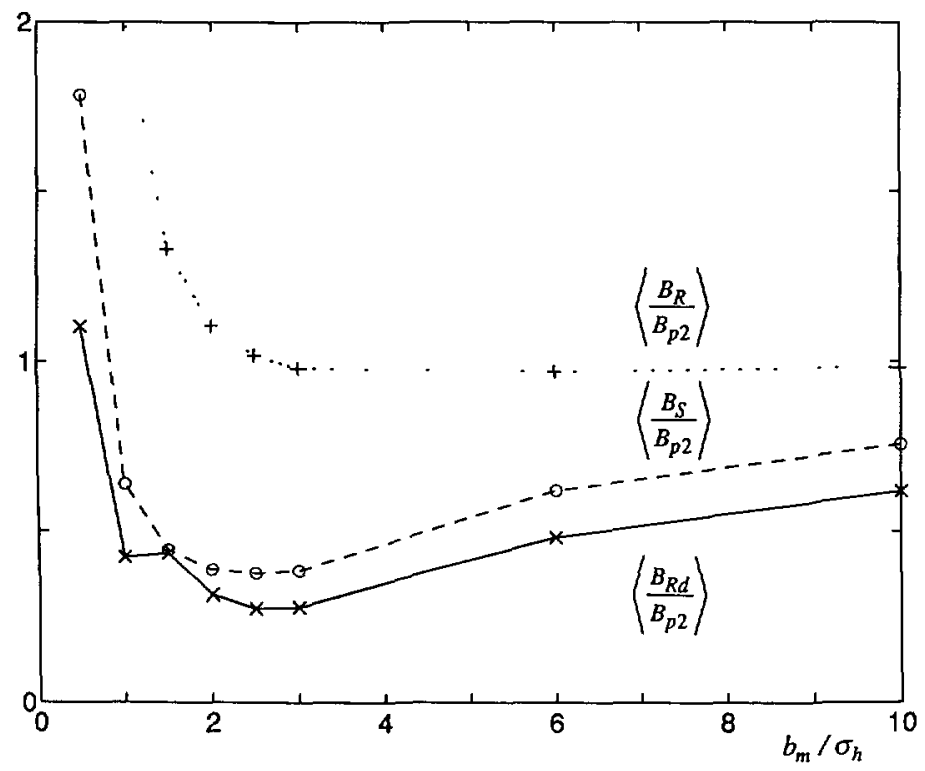

Fig. 11. - Dimensionless permeabilities $\left\langle B_{\mathrm{Rd}} / B_{\mathrm{p}_{2}}\right\rangle$ (solid lines), $\left\langle B_{\mathrm{S}} / B_{\mathrm{P}_{2}}\right\rangle$ (dashed lines), and $\left\langle B_{\mathrm{R}} / B_{\mathrm{p}_{2}}\right\rangle$ (dotted lines) as functions of the mean separation $b_{\mathrm{m}} / \sigma_{h}$ for self-affine fractures. Data are for $\theta=0$ and $\zeta=08$.

The ratio $\left.<B_{\mathrm{Rd}} / B_{\mathrm{S}}\right\rangle$ is closer to 1 than $\left\langle B_{\mathrm{R}} / B_{\mathrm{S}}\right\rangle$. The analysis of all the numerical data showed that $b_{\mathrm{Rd}}$ is a good approximation for $b_{\mathrm{S}}$ when $b_{\mathrm{m}} / \sigma_{h}$ is larger than 2 for uncorrelated surfaces $(\theta=0)$ and when $b_{\mathrm{m}} / \sigma_{h}$ is larger than 1 for highly correlated surfaces $(\theta=0.9)$.

It is found that if $b_{\mathrm{m}} / \sigma_{h}$ and $l / \sigma_{h}$ become large enough $\left(b_{\mathrm{m}} / \sigma_{h}>7, l / \sigma_{h}>5\right)$, the ratio $B_{\mathrm{R}} / B_{\mathrm{S}}$ tends toward 1 and the solution of the Reynolds equation with $b$ defined in (5a) is a good approximation to the Stokes solution. If one of these parameters, $b_{\mathrm{m}} / \sigma_{h}$ or $l / \sigma_{h}$, is smaller than these limit values, the ratio $B_{\mathrm{R}} / B_{\mathrm{S}}$ becomes larger than 1 .

These results are relative to Gaussian fractures which are characterized by the correlation length $l$; it is seen from Figure 7 that the fracture permeability is only slightly influenced by the correlation parameter $\theta$.

These computations were extended to self-affine surfaces since they are representative of real 


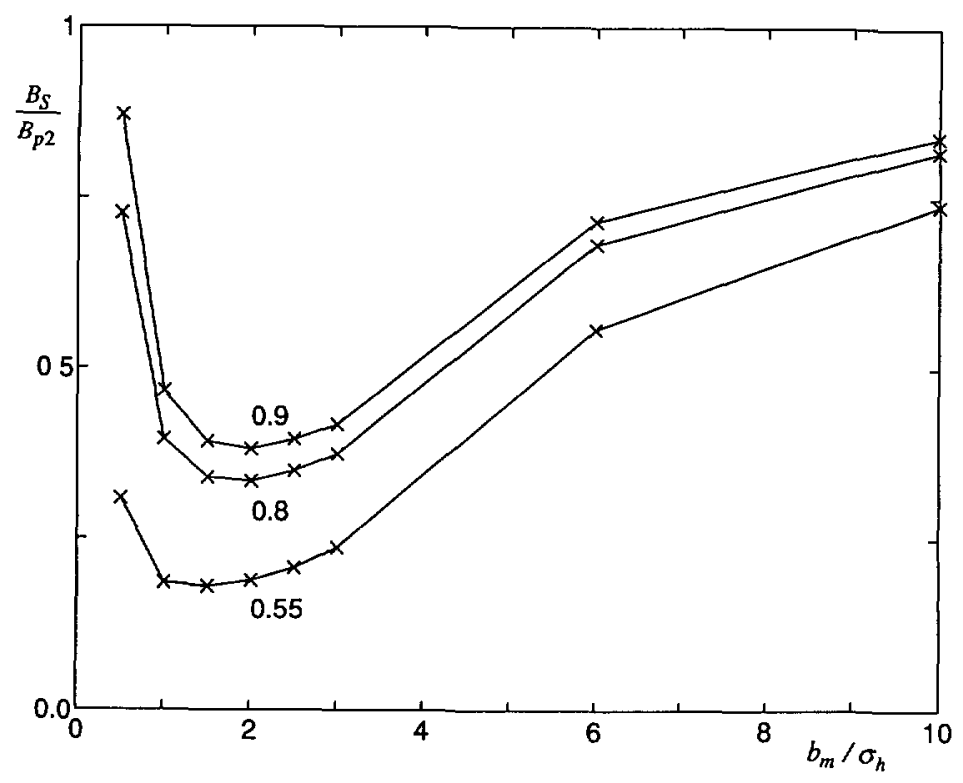

Fig. 12. - Dimensionless permeabilities $B_{\mathrm{S}} / B_{p_{2}}$ as functions of the mean separation $b_{\mathrm{m}} / \sigma_{h}$ for selfaffine fractures with different $\zeta$. Data are for: $\theta=0$.

surfaces. Figure 11 shows mean permeabilities calculated for 5 realizations of uncorrelated selfaffine surfaces with a roughness exponent $\zeta=0.8$. It is useful to compare Figure 11 with Figure 8; the general shape of the curves is the same for Gaussian and self-affine surfaces. However, the permeability for self-affine surfaces is always larger than that of Gaussian surfaces; this may be related to the fact that the characteristic roughness length $l$ is smaller for Gaussian surfaces than for self-affine surfaces where it is of the order of the total size of the sample because of the upper cut-off. The Reynolds equation does not provide a better approximation to permeability than before. However, the use of $b_{\mathrm{Rd}}$ instead of $b$ yields a considerable improvement of the Reynolds approximation.

The influence of the roughness exponent $\zeta$ was analysed for the same realization of fracture surfaces (Fig. 12). It was found that $B_{\mathrm{S}}$ and $B_{\mathrm{Rd}}$ are decreasing functions of $\zeta$, while $B_{\mathrm{R}}$ is insensitive to it; this finding is in good agreement with the numerical results of [2].

\section{Conclusions}

Fluid flow within fractures with randomly generated surfaces with Gaussian correlations was numerically studied using Stokes and Reynolds equations. Taking the solution of the Stokes equation as the reference, it was found that the Reynolds equation based on the aperture $b$ (5a) overestimates the permeability by a factor larger than 2 in the range, $l / \sigma_{h}<3$ and $b_{\mathrm{m}} / \sigma_{h}<3$. The ratio $B_{\mathrm{R}} / B_{\mathrm{S}}$ varies slowly with $l$ in an intermediate range, but grows sharply as $l / \sigma_{h}$ tends to 1 . This behavior is unaffected by a correlation between the fracture surfaces. The use of the aperture $b_{\mathrm{d}}$ in the Reynolds equation provides a much better approximation to $B_{\mathrm{S}}$, which underestimates it.

This analysis was extended to fractures with self-affine correlations; the conclusions are basically the same for the validity of the approximation of the permeability by the Reynolds 
equation. However, the use of the aperture $b_{\mathrm{d}}$ provides a better improvement than in the previous case.

This paper may be concluded by noting that real fractures may be much more complex than the idealization presented here. Most real fractures are found to contain fracture filling materials, etc., intersections and other perturbations that cause the proposed model to be generally incomplete. Moreover, the stationarity of the random fields is questionable. However, all these additional difficulties are better addressed by a local analysis of the velocity field by means of the Stokes equations

\section{Acknowledgments}

This work was partially supported by a grant from Ecotech. Part of the computations were performed at CNUSC.

\section{References}

[1] Tsang Y.W., and Tsang C.F., Wat. Res Res. 25 (1989) 2076.

[2] Brown S R., J. Geophys. Res. 92 (1987) 1337.

[3] Zimmerman R.W., Kumar S. and Bodvarsson G.S., Int J. Rock Mech Min. Sct. Geomech Abstr. 28 (1991) 325.

[4] Hasegawa E., and Izuchi H, Bull JSME 26 (1983) 514.

[5] Pozrikidis C., J. Fluıd Mech. 180 (1987) 495.

[6] Koplik J., Ippolito I. and Hulın J.P, Phys Fluıds A5 (1993) 1333.

[7] Ippolito I., Daccord G, Hinch E.J. and Hulin J.P., J Contam Hydrol. 16 (1994) 87.

[8] Adler P.M , Porous Medıa: Geometry and Transports (Butterworth/ Heınemann, Stoneham, MA, 1992).

[9] Thovert J.F., Salles J. and Adler P.M., J. Microsc 170 (1992) 65.

[10] Brown S.R and Scholz C.H., J Geophys Res. 91 (1986) 4939.

[11] Gentier S, Morphologie et comportement hydromécanique d'une fracture naturelle dans le granit sous contrainte normale, Thesis, Université d'Orléans, France (1986).

[12] Brown S R, Kranz R.L. and Bonner B P., Geoph Res. Lett. 13 (1986) 1430.

[13] Brown S.R. and Scholz C.H., J Geophys. Res 90 (1985) 12575.

[14] Hansen A., Hinrichsen E.L. and Roux S., Phys. Rev Lett. 66 (1991) 2476.

[15] Måløy K.J., Hansen A., Hinrıchsen E L. and Roux S , Phys. Rev Lett. 68 (1993) 213.

[16] Lemaitre R. and Adler P.M , Trans. Porous Medıa 5 (1990) 325.

[17] Zimmerman R.W., Chen D.W. and Cook N G.W., J Hydrol 139 (1992) 79.

[18] Henriette A., Jacquin C.G and Adler P.M., Phys Chem. Hydrodyn. 11 (1989) 63.

[19] Yao J., Modélisation numérique de la porosité et de la perméabilité des roches, appliquée à la compréhension des problèmes d'injectivité dans les formations argilo-gréseuses, Thesıs, Université de Paris - VI (1995) in preparation.

[20] Abelin H., Birgersson L., Gidlund J. and Neretnieks I., Wat. Res Res. 27 (1991) 3107.

[21] Moreno L, Tsang Y.W., Tsang C.F., Gale F.V. and Neretnieks I., Wat. Res. Res. 34 (1988) 2033 